\title{
Study of capabilities of making detection device for cerebral ischemic state
}

\author{
Lobekin VN, Petrov RV, Bichurin MI*, Rebinok AV and Sulimanov RA \\ Novgorod State University, Russia
}

\begin{abstract}
Problems and causes of brain ischemia have been considered. Relevance of the given problem has been revealed, considering the fact that, on average, the incidence of acute brain circulation disorders of ischemic type makes up to $3,5 \%$ of population. The problem may be solved by the way of dopplerography which allows examining speed and direction of blood flow. The characteristic of this method is that measurement is performed at contralateral extra-intracranial periorbital arterial anastomoses at the level of angular and supratrochlear arteries which pass near each eye, thus, the duration of examination shortens. The important feature of performance of anastomoses of extracranial level is that they can be activated extremely quickly to compensate brain hypoxia; these are the so-called true group of anastomoses. Techniques and devices to diagnose the condition of brain have been pointed out. The variant of a mobile and portable device to detect ischemic state of brain has been suggested. The device works due to Doppler effect, and sensors are positioned to measure accurately the speed and direction of blood flow. Advantages of the given device are the examination of ischemic condition of affected visual organs and brain, as well as the analysis of both blood speed and direction.
\end{abstract}

\section{Introduction}

Worldwide, the diseases of cardiovascular system are the leading causes of death due to morbidity. Among these diseases, cerebrovascular pathology takes a significant proportion, leading to brain infarctions with clinical manifestations of neurological deficiency - stroke. This problem is urgent because the incidence of the condition and resultant disability increase among persons of middle and young (employable) age. The given problem was discussed in detail in publications [1].

Disorders of brain circulation are one of the main causes of mortality in developed countries. Effective prevention and treatment of brain circulation disorders caused by pathologic winding of carotid arteries, atherosclerotic injury of arteries, various occlusions, and stenosis often depend on detection of bloodstream parameters. Current examination techniques of brachiocephalic arteries and brain blood flow, such as digital subtraction angiography, computer tomography angiography, magnetic resonance imaging, are all invasive and/or unsafe for patients, expensive, and mainly give information about morphologic changes, but do not allow evaluating quantitative characteristics of bloodstream particularly. The problem of creating a detection device for ischemic state of brain becomes urgent and requires investigation. The device refers to the field of medicine, namely functional diagnostics, neurosurgery, neurology, and can be used to diagnose the ischemic cerebral state.

\section{Cerebral ischemia}

\section{General information}

Ischemia of brain is the disease occurring due to cerebral hypoxia caused by poor blood supply of brain tissues. The disease produces numerous symptoms related to brain function impairment: retarded information perception and decision making, speech and coordination disorders, vision impairment, etc. At the onset, symptoms are latent, but, as the disease progresses, become evident. Quick fatigue, sleeplessness, frequent headaches, bad memory may be the symptoms of ischemic disease of brain vessels (circulation disorders). The disease may be acute or chronic.

Atherosclerosis, thrombi formation, heart failure, hypertension are main factors of cerebral ischemia. It is very important to diagnose this severe disease early. Diseases provoking ischemia must not be neglected. Complications can lead to disability and even to fatal outcome. Additional causes of cerebral ischemia:

- cardiovascular diseases affecting central hemodynamics;

- blood diseases leading to its viscosity;

- various angiopathies - diseases affecting blood vessel walls;

- diabetes mellitus;

- obesity;

- carbon monoxide poisoning;

- anemia;

- old age;

- heredity;

- smoking.

The average incidence of acute cerebral circulation disorder of ischemic type is $12-35$ people out of 1000 . According to recorded data of strokes, about 400000 strokes are recorded in Russia annually, and about 320000 of them belong to ischemic type. In the US, Europe and

*Correspondence to: Bichurin MI, Novgorod State University, Veiky Novgorod 173003, Russia, E-mail: mirza.bichurin@novsu.ru

Received: July 05, 2018; Accepted: July 24, 2018; Published: July 27, 2018 
Australia the first stroke occurs at age over 45 , about 400 people out of 100000 are affected.

Structure of occluding events of cerebral vascular system leading to brain infarctions:

a) atherosclerotic stenosis and thrombosis - $84 \%$,

6) pathologic winding and twisting of main arteries of neck - $8 \%$,

в) extravascular compressions - $4 \%$,

г) thromboembolism - 4\% [1,2].

During the examination of brain matter perfusion parameters (a CT-perfusion method) the following results were revealed:

- In patients with bilateral, hemodynamically significant, stenosis of internal carotid artery (over 60\%) the indices of brain perfusion deviated mainly bilaterally.

- In patients with lateral, hemodynamically significant, injuries of mentioned arteries, the indices of blood flow in hemisphere ipsilaterally to stenosis, in closed circle of Willis, deviated insignificantly in relation with contralateral hemisphere.

- In patients with lateral, hemodynamically significant, injuries of mentioned arteries, the indices of blood flow in hemisphere ipsilaterally to stenosis, in opened circle of Willis, showed that the blood flow in basins ipsilaterally to stenosis decreased significantly, varying much compared with contralateral basin [3].

Among pathologic cerebral hemodynamic events, acute thrombosis and embolism should be distinguished, as well as the group of narrowing events, which progress slowly and may be revealed at an early stage of developing. Another important pathologic phenomenon of transient functional occlusion in a narrowed vessel should be noted, that occurring at accelerated blood flow, e.g. in increased arterial pressure (Figure 1). Thus, it was demonstrated that arterial stenosis exceeding $50-55 \%$ of an artery's initial diameter influenced negatively the blood flow.

Stenosis is considered to be dramatic, when an artery is narrowing up to $70-80 \%$, since then the turbulent motion of blood flow occurs, and the volume of blood decreases, but compensation due to increased heart work becomes impossible. Thus, in elderly persons with marked changes of heart activity, it is atherosclerotic stenosis but not cerebral arterial occlusion that leads to cerebral infarction, producing clinical stroke in $70 \%$ of all cases. Then, in narrowed occluded parts of arteries, the blood flow slows down abruptly, leading to aggregation of formed blood elements and microembolism. Some patients may acquire even retrograde bloodstream leading to turbulence once more and increasing the risk of thrombi formation.

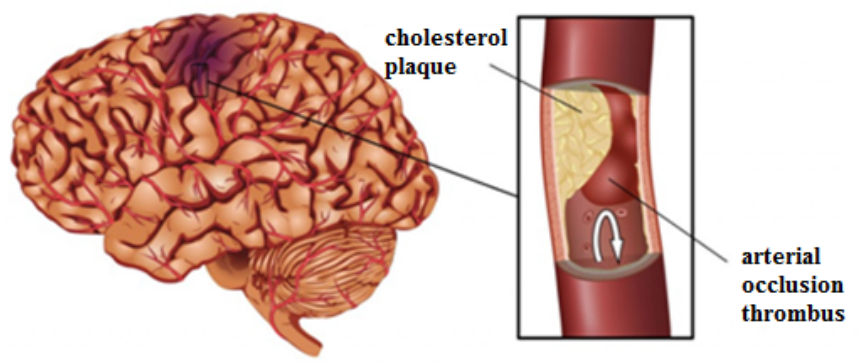

Figure 1. Occurrence of cerebral ischemia: cholesterol plaque, thrombus occluding artery
To determine ischemic stroke, some general criteria and notions were established: response of brain to progressive chronologic cerebral circulation decrease, "nuclear" ischemic zone, ischemic penumbra functionally altered, but vital and potentially restorable, brain region, i.e. parabiosis zone. Time factor is determinant during the transformation of penumbra zone into cerebral infarction (necrosis). In other words, in clinical stroke there is a brief (about 4 hours) "therapeutic window" when medical intervention can lessen infarction zone, fight brain edema, and correct vascular and metabolic reactions [4].

\section{Cerebral hemodynamics}

Cerebral hemodynamics is a dynamic system with many complex protective and adaptive mechanisms as at the level of macro-organism as at that of local vascular net in the region of head and neck, characterized by plasticity of metabolic processes in nervous tissues.

Normally, at rest, about $20 \%$ of all blood circulating around the organism pass through the vascular system of brain.

Main arteries of head and neck perform both transport function and cerebral hemodynamics regulation, especially when any cerebrovascular pathology is present, forming and turning on extraintracranial arterial anastomoses. Then hemodynamics replaces into the area of less pressure, until retrograde blood flow occurs. Main arteries of brain are shown in figure 2. Cerebral arteries, their branches and anastomoses can actively change their lumen, thus regulating and distributing blood flowing to the brain [5].

To maintain a normal brain function, oxygen tension in brain matter must be within $20 \mathrm{~mm}$ of mercury, this variable corresponds to brain oxygen intake in the amount of $3,3 \mathrm{ml}$ per $100 \mathrm{~g}$ of cerebral matter in a minute. The decrease of this variable to $2,7 \mathrm{ml}$ is the evidence of brain hypoxia. Oxygen supply as mentioned is possible when the intensity of brain circulation is equal to about $55 \mathrm{ml}$ of blood per $100 \mathrm{~g}$ of brain matter in a minute. These numbers are characteristic of normal regional cerebral blood flow. They may change within $40-75 \mathrm{ml} / 100$ $\mathrm{g} / \mathrm{min}$. The bloodstream decrease below $40 \mathrm{ml} / 100 \mathrm{~g} / \mathrm{min}$ points at blood supply deficiency. Until recently, thrombosis and embolism were considered to be main limiting factors of cerebral blood supply. At the same time, alterations associated with stenosis of vascular canals of brain were underestimated. Experimental and clinical studies revealed certain patterns of blood flowing in a narrowed vessel, mechanisms and conditions of its compensation, as well as the dependence of a variety of bloodstream parameters on the degree, shape and extension of stenosis. The probability of temporary functional occlusion in a narrowed vessel at the height of bloodstream acceleration was proven in high blood pressure as well. So, arterial stenosis exceeding $50 \%$ of initial diameter was shown to have the negative effect on bloodstream. The narrowing of an artery to $70-80 \%$ is considered to be critical. Such size of stenosis affects not only the bloodstream, - there occurs the state of unstable flow or turbulent motion, the whole volume of blood decreases, and the hard work of heart cannot compensate it [6].

\section{Techniques and devices diagnosing ischemic condition of brain}

The brain connects functions of bodily organs and systems, coordinating them. Under its supervision, the information given by sense organs is perceived and discriminated, movements are coordinated, memory and attention can function properly, speech can be formed and "decoded". Since the brain is the most complex organ of the human body, it requires the most sophisticated methods for its investigation. 


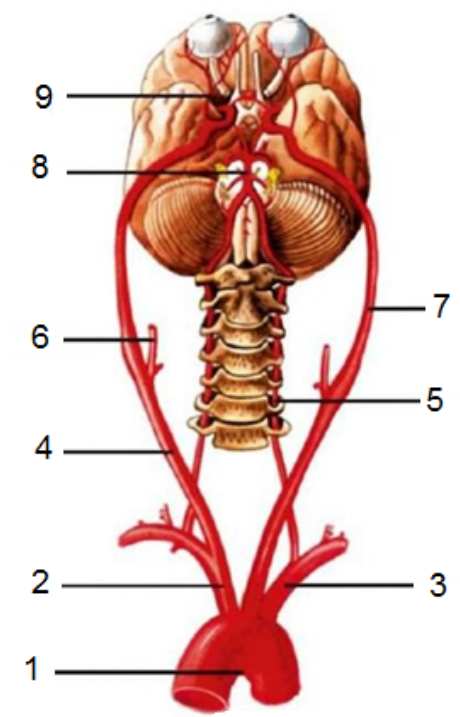

Figure 2. Main arteries of brain. 1 - arch of aorta; 2 - brachiocephalic trunk; 3 - left subclavicular artery; 4 - right common carotid artery; 5 - vertebral artery; 6 - external carotid artery; 7 - internal carotid artery; 8 - basilar artery; 9 - ophthalmic artery

Brain diseases disturb its proper functioning, resulting in disorders of various parts of the body. The commonest of them are:

- Alzheimer's disease which occurs when the organism produces a pathologic protein leading to atrophy of nerve cells,

- Cerebral stroke, i.e. an acute circulation disorder affecting tissues and body functions,

- Tumor, i.e. the growth of cells non-typical for the brain which provoke the increase of intracranial pressure,

- Epilepsy, characterized by recurrent disorders of brain functioning. During the seizure, a person loses consciousness, convulsions may occur.

Early diagnosis can reveal diseases and their causes, thus making them treatable. Let us consider main methods of brain examination:

1. EchoEG (echoencephalography) - The ultrasound diagnosis of brain pathologies performed with oscilloscope. The device fixes a reflected ultrasound and reflects the returned signal on the display. EchoEG is indicated, suspecting a tumor or craniocerebral trauma.

2. USDG (ultrasound dopplerography) - The examination performed to evaluate the bloodstream in major and medium-sized vessels of head and neck. Ultrasound dopplerography may be used to detect the first signs of vascular channel pathologies as well as to control the treatment being performed. Dopplerography examination takes into account the changes of frequency of ultrasound waves reflected from movable structures of the body. The examination is highly informative way of obtaining data about functional activity and condition of vessels.

3. REG (rheoencephalography) of brain - The way of evaluation of functional condition of vessels based on recording of the changes of total electrical resistance of tissues and conditioned by pulse fluctuations of vessels. Rheoencephalography is used to diagnose tumors, head traumas, epilepsy, migraines.

4. MRI (magnetic resonance imaging) of brain vessels - The complicated, but informative, way of examination based on the mechanism of nuclear magnetic resonance.
5. MRA (magnetic resonance angiography) - The way which does not require the direct puncture of an artery, progressing, and very promising in the examination of a vascular channel. MRA allows making three-dimension reconstruction of the vascular net in the region examined, as well as examining certain nervous vessels and trunks isolated from the projection of brain sections.

6. EEG (electroencephalography) - The way of recording fluctuations of electrical potentials of the brain with electroencephalograph. Electrodes attached to the head read bioelectrical currents of the brain fixed on the paper or reflected on the display. The procedure is indicated in cases of retardation of psychologic and speech development, traumas, epileptic seizures.

7. NSG (neurosonography) - The ultrasound examination of the brain of newborns until the anterior fontanel is closed. Neurosonography is safe and very informative, detecting pathologies at early stages and evaluating the effectiveness of the treatment performed.

8. CT (computer tomography) (Figure 3) of brain vessels - The way which measures how intensive X-rays are, when passing through cerebral tissues. The way allows viewing the sections of the brain at different levels of horizontal plane.

9. PET (positron emission tomography) - The way of using radiopharmaceuticals which allow making three-dimension reconstruction of functional processes of the brain $[7,8]$.

\section{Capabilities of making a device to detect cerebral ischemic condition}

\section{Using of function of anastomoses}

Normally, cerebral vascular anastomoses are inactive. They can be activated mainly to perform vascularization (blood reflow) of the vascular basin of the brain with deficient blood supply. There may be temporary dynamic factors, e.g. extension or lateroflexion in the cervical department of spine with compression of vertebral or carotid arteries. Then, the most important vascular system of cerebral anastomoses - circle of Willis - starts compensating cerebral hemodynamics towards decreased intra-arterial pressure. The important feature of the compensation is that anastomoses turn on extremely quickly to realize mechanisms of compensation of cerebral hypoxia, the so-called true group of anastomoses.

It should be noted that a "classical" (closed) type of structure of circle of Willis occurs only in about $30-50 \%$ of all cases, but various anomalies of its structure are up to $82 \%$. Several morphological levels of collateral circulation are distinguished [9].

Extracranial level is the group of anastomoses between the basin of carotid arteries and that of subclaviculovertebral ones. The most important of them are: anastomoses between occipital artery (the basin of external carotid artery) and muscular branches of vertebral artery, between occipital artery (the basin of external carotid artery)

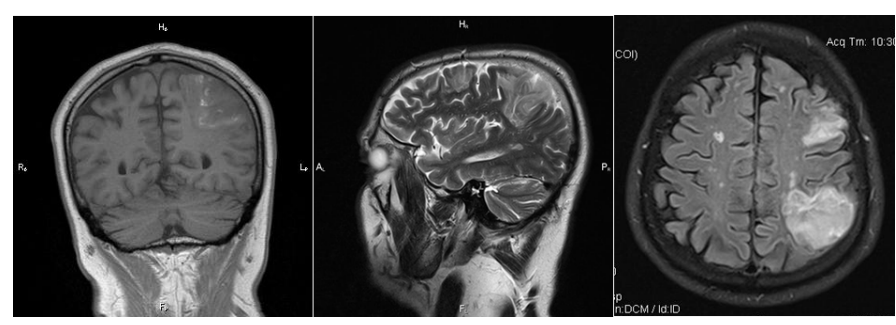

Figure 3. Computer tomography 
and arteries of thyrocervical and costocervical trunks (the basin of subclavicular artery), between superior thyroid arteries (the basin of external carotid artery) and inferior thyroid arteries (the basin of subclavicular artery). Also, the last anastomosis connects heterolateral basins of carotid and subclavicular arteries. Anastomoses between the basins of right and left external carotid arteries at the level of lingual and external maxillary arteries were found.

Besides above-mentioned, there is a variety of other anastomoses. When the internal carotid artery is closing, the most significant is the direct extra-intracranial anastomosis at the level of the branches of ophthalmic artery with the branches of external carotid artery in the projection of superior medial angle of orbit, nasal dorsum and frontal region [10].

Branches of right ophthalmic artery are shown in figure 4. Supratrochlear artery, a. supratrochlearis, - one of the terminal branches of ophthalmic artery located inside from supraorbital artery. It circumflexes supraorbital margin, and ascending, supplies the skin of medial forehead and muscle. Its branches anastomose with those of contrary-side artery of the same name. Dorsal nasal artery, a. dorsalis nasi, is, like supratrochlear artery, the terminal branch of ophthalmic artery. It goes ahead, lying above medial palpebral ligament, branches towards lacrimal sac, and comes to nasal dorsum. Here it connects with angular artery (the branch of a. facialis), thus forming the anastomosis between the systems of internal and external carotid arteries [11]. Location of facial arteries are shown in figure 5.

\section{Supposed variant of the device designed to detect ischemic condition of brain}

The invention refers to the field of medicine, namely functional diagnostics, neurosurgery, and neurology, and may be used to measure the speed of bloodstream in vessels by the way of dopplerography.

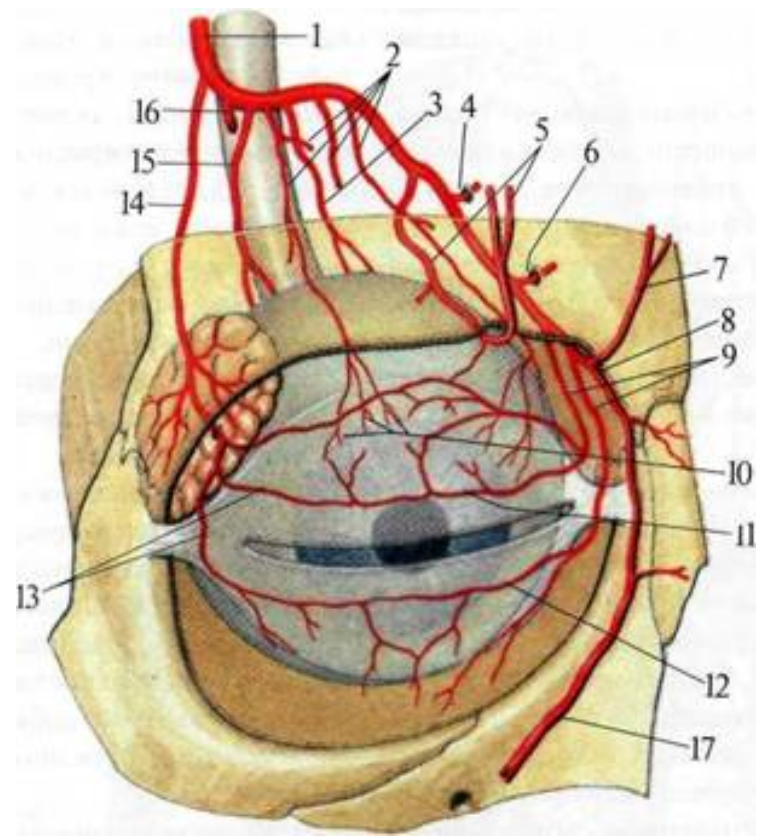

Figure 4. Branches of right ophthalmic artery (semi-schematically). $1-$ a. ophthalmica; 2 - aa. musculares; 3 - a. ciliaris longa; 4 - a. ethmoidalis posterior; 5 - a. supraorbitalis; $6-$ a. ethmoidalis anterior; $7-$ a. supratrochlearis; $8-$ a. dorsalis nasi; $9-$ aa. palpebrales mediales; 10 - aa. episclerales; 11 - arcus palpebralis superior; 12 - arcus palpebralis inferior; 13 - aa. palpebrales laterales; $14-$ a. lacrimalis; $15-$ a. ciliaris posterioris brevis; $16-\mathrm{a}$. centralis retinae; $17-\mathrm{a}$. angularis

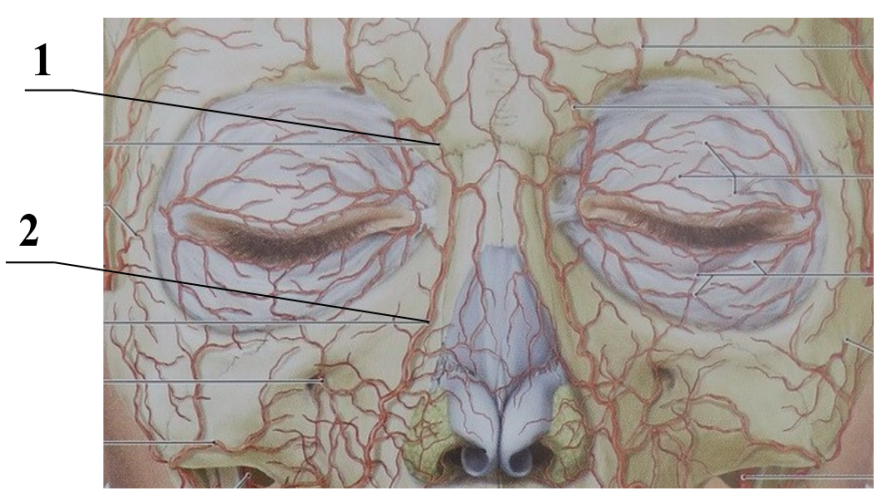

Figure 5. Location of facial arteries. 1 - dorsal nasal artery; 2 - angular nasal artery

Herewith, the measurement is done at contralateral extra-intracranial periorbital arterial anastomoses of the brain at the level of angular and supratrochlear arteries passing near each eye. The speed of blood is measured in each anastomosis. Then parameters of blood speed and direction measurement in the right anastomosis are compared with those in the left anastomosis. Then the data are compared with normal parameters. If the speed and direction are different in the right and left anastomoses and don't meet normal general or individual parameters, the signs of cerebral ischemia are diagnosed.

The problem is solved thanks to dopplerography which is the way of measuring blood speed and determining blood direction. It allows examining at contralateral extra-intracranial periorbital arterial anastomoses of the brain, at the level of angular and supratrochlear arteries passing near each eye, and thanks to that fact the duration of examination shortens. The examination is performed by locating the vessel at an angle $\left(30-40^{\circ}\right)$, taking into account the anatomical characteristics of vessel location. The measurement of bloodstream speed and direction is performed. Then the parameters of blood speed and direction in the right anastomosis are compared with those in the left anastomosis. Then the data obtained are compared with normal parameters. If the speed and direction of bloodstream are different in each anastomosis, and do not correspond to normal general or individual parameters, then the signs of cerebral ischemia are diagnosed, thus the diagnosis may be adequate. Generally, a physiological norm in adult patients is $50-55 \mathrm{ml}$ of blood per $100 \mathrm{~g}$ of brain matter. Also there may exist an individual norm of a person, detected by preliminary measurement of initial hemodynamics. Further, the individual norm may be used to compare the results of examination. The given observation and measurement of bloodstream speed and direction in the brain may be used to diagnose ischemic cerebral condition by noninvasive way. The advantage of the way is that it allows performing preventive measures against cerebral ischemia developing, as the given way records the very onset of the process. The way gives opportunity to make a mobile and portable device which may be carried by a patient for a long time. The device can monitor a patient's brain blood flow for a long time in various physical and psycho-emotional conditions outside the hospital.

To obtain such technical results, it is suggested to make the device functioning with Doppler effect. The device allows positioning sensors in the pre-established position to measure bloodstream speed and direction accurately. The device presents a measuring and computing complex. It consists of an attachment construction for head resembling an eyeglass frame, a construction designed to attach and adjust ultrasound sensors, a measuring unit with ultrasound emitting 


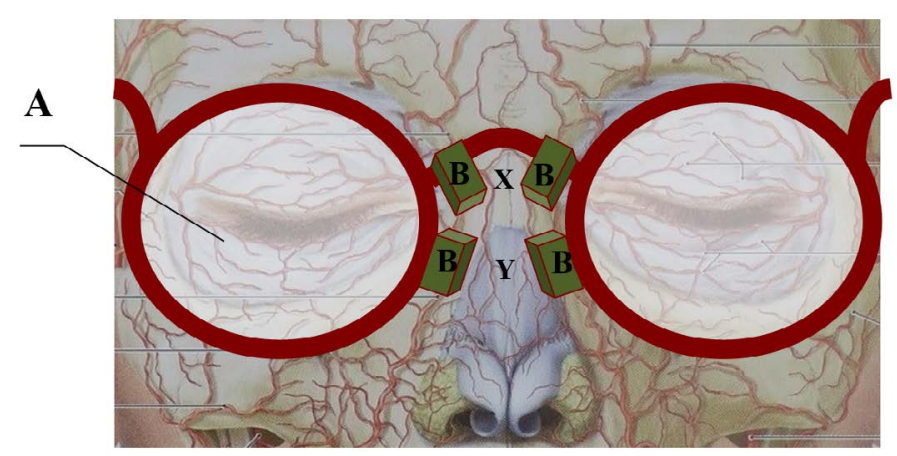

Figure 6. Device scheme. A - construction resembling eyeglass frame; B - dopplerography ultrasound sensor with radio transmitter; $\mathrm{X}$ - sensor position for angular artery; $\mathrm{Y}$ - sensor position for dorsal artery

sensors, and an electronic unit including signal generating circuit, signal processing circuit, display, and signal transmitter. As anatomical parameters of face and the location of arteries may be different in different people, the system of sensor positioning is proposed to be adjusted according to the individual characteristics of a person. Performance capabilities of the given device include monitoring of ischemic condition as of affected visual organs as of brain, analysis of both blood speed and direction, high accuracy of examination due to measurement of two anastomoses with two dopplerography sensors. Device scheme is shown in figure 6.

\section{Conclusion}

The given paper studies capabilities of making the device to diagnose ischemic condition of brain. Signs and causes of cerebrovascular pathology have been considered. Techniques and devices for cerebral condition diagnostics have been studied and analysed. We revealed that, to prevent the development of cerebral ischemia, it was necessary to work out the non-invasive way of measuring blood speed and direction. The given way allows making a long-term mobile portable device intended for diagnosing in out-clinical setting.

\section{Acknowledgement}

The paper was prepared with financial support of Ministry of Education and Science of Russia within the framework of the state task, project №8. 11100. 2018/11. 12.

\section{References}

1. Vereshchagin NV (2004) Heterogenety of stroke in clinical practice. Atmos Nervn Bolezni 1: 19-20

2. Shmidt EV (1963) Stenosis and thrombosis of carotid arteries and cerebral circulation disorders. M Medgiz: 319.

3. Amelin ME, Shevchenko AV, Kurbatov VB, Grishkov AV, Surmava AE, et al. (2007) Evaluation of brain circulation in patients with stenoses of internal carotid artery by CT-perfusion method. J Angiol Vascular Surg 4.

4. Odinak MM, Voznuk IA, Golohvastov SY, Fockin VA (2005) Monitoring of perfusion disorders at extremely acute stage of ischemic stroke. Bulletin of Russian Military Medical Academy? 2: 25-30

5. Gannushkina YV (1973) Collateral circulation in brain. M Medicina: 253

6. Shmidt EV, Lunev DK, Vereshchagin NV (1976) Vascular diseases of brain and spinal cord. M Medicina: 284

7. Trufanov GE, Fokin VA, Piyanov IV, Bannikova EA (2005) Radiographical computer and magnetic resonance tomography in diagnostics of ischemic stroke. St. Petersburg. Elbi-SPb: 191.

8. Ginsberg MD, Pulsinelli WA (1994) The ischemic penumbra, injury thresholds, and the therapeutic window for acute stroke. Ann Neurol 36: 553-554. [Crossref]

9. Vereshchagin NV, Morgunov VA, Gulevskaya TS (1997) Pathology of brain in atherosclerosis and arterial hypertonia?

10. Gulevskaya TS, Morgunov VA (2009) Pathological anatomy of disorders of cerebral circulation in atherosclerosis and arterial hypertonia? Medicina: 296.

11. Akimov GA (1974) Transient disorders of cerebral circulation. L Medicina: 168

Copyright: $@ 2018$ Lobekin VN. This is an open-access article distributed under the terms of the Creative Commons Attribution License, which permits unrestricted use, distribution, and reproduction in any medium, provided the original author and source are credited. 